IdeAs

Idées d'Amériques

$10 \mid 2017$

États-Unis / Cuba : une nouvelle donne?

\title{
Lawrence Ross, Blackballed. The Black and White Politics of Race on America's Campuses
}

New York, St. Martin Press, 2016, 232 p.

\section{Clémentine Berthélémy}

\section{OpenEdition}

\section{Journals}

Édition électronique

URL : https://journals.openedition.org/ideas/2179

DOI : 10.4000/ideas. 2179

ISSN : 1950-5701

Éditeur

Institut des Amériques

Référence électronique

Clémentine Berthélémy, «Lawrence Ross, Blackballed. The Black and White Politics of Race on America's Campuses », IdeAs [En ligne], 10 | 2017, mis en ligne le 18 décembre 2017, consulté le 19 octobre 2022. URL : http://journals.openedition.org/ideas/2179 ; DOI : https://doi.org/10.4000/ideas.2179

Ce document a été généré automatiquement le 19 octobre 2022.

\section{c) (하 $\ominus$}

Creative Commons - Attribution - Pas d'Utilisation Commerciale - Pas de Modification 4.0 International - CC BY-NC-ND 4.0

https://creativecommons.org/licenses/by-nc-nd/4.0/ 


\section{Lawrence Ross, Blackballed. The Black and White Politics of Race on America's Campuses}

New York, St. Martin Press, 2016, 232 p.

Clémentine Berthélémy

\section{RÉFÉRENCE}

Lawrence Ross, Blackballed. The Black and White Politics of Race on America's Campuses, New York, St. Martin Press, 2016, 232 p.

1 Le 7 mars 2015, à l'occasion du cinquantième anniversaire de la marche civique de Selma, Barack Obama déclare que l'Amérique «porte toujours l'ombre de son histoire raciale $»^{1}$. Seulement quarante-huit heures plus tard, un scandale impliquant les membres d'une fraternité de l'Université d'Oklahoma atteste de la véracité des propos du président. Dans une vidéo publiée en ligne, les membres de la prestigieuse fraternité Sigma Alpha Epsilon entonnent à l'unisson un chant raciste prônant le lynchage des Noirs : « Il n'y aura jamais de nègres [dans ma fraternité], vous pouvez le pendre à un arbre, mais jamais son nom ne sera collé au mien » (p. 8). Cet épisode n'a en fait rien d'inédit puisqu'il s'ajoute une vaste liste d'incidents racistes recensés sur l'ensemble des campus universitaires. Face à l'incompréhension et l'embarras que suscite la recrudescence de ce genre d'incidents, l'ouvrage Blackballed de Lawrence Ross vient à point nommé.

2 Conférencier, journaliste et romancier américain, Lawrence Ross entend lever le voile sur « l'un des lieux de vie les plus racialement hostiles des États-Unis » (p. 5) : le campus universitaire. Plus exactement, l'ouvrage fait la lumière sur les zones d'ombre du système $\operatorname{grec}^{2}$ dit "traditionnellement blanc" au sein duquel sévit un racisme endémique soutenu par l'existence d'un "privilège blanc (...) codifié » (p. 51). Cette approche tout à fait pertinente - quoique quelque peu restreinte - s'inscrit dans la 
continuité des travaux de Lawrence Ross exposés en 2000 dans The Divine Nine - sans nul doute le plus important ouvrage consacré aux fraternités et sororités étudiantes afro-américaines. L'intérêt de Blackballed répond à un triple objectif : reconnaître l'existence d'une crise raciale majeure sur les campus ; mettre en évidence le rôle joué par les universités dans le développement d'un environnement racialement hostile; et enfin imaginer des solutions adaptées et efficaces pour rendre les campus plus accueillants. Blackballed s'ordonne en sept chapitres selon un rythme dynamique et une vivacité de ton particulière conférant à l'ouvrage l'un de ses principaux attraits.

3 L'incident de l'Université d'Oklahoma constitue le point de départ de l'ouvrage. L'intérêt de ce premier chapitre n'est pas tant d'informer le lecteur sur les circonstances précises de l'incident mais plutôt de le lui présenter comme une manifestation symptomatique d'un environnement où le privilège blanc et le racisme perdurent. Comme dans la suite de l'ouvrage, Lawrence Ross ne se contente pas ici d'un simple assemblage des faits. En effet, il apporte d'emblée un éclairage historique aussi précieux que captivant sur la façon dont les fraternités traditionnellement blanches ont usé, depuis la fin des années 1920, de clauses restrictives visant à interdire l'accès de leurs confréries aux étudiants afro-américains. En 1928, plus de la moitié d'entre elles avait en effet mis en place ces restrictions, facilitant ainsi l'émergence de fraternités non-traditionnelles (notamment afro-américaines). Nonobstant l'élimination progressive de ces clauses restrictives au milieu des années 1960, le système grec est aujourd'hui encore marqué par une ségrégation de facto. Pour Lawrence Ross donc, « rien n'a véritablement changé » (p. 47).

4 Pour le prouver, l'auteur met à profit son expérience du terrain et du système grec ${ }^{3}$ au cours des trois chapitres suivants (2-4). Il examine des cas précis de manifestation du privilège blanc ainsi que leur effet exclusionnaire, lesquels sont corroborés par des témoignages, souvent poignants, d'étudiants et alumni afro-américains. À cet égard, on notera l'originalité et la pertinence des exemples choisis. Le plus frappant est sans conteste celui de l'Université de l'Alabama (chapitre 2) où une coalition secrète d'étudiants - semblable aux Skull and Bones de Yale - exerce depuis plus d'un siècle un pouvoir politique des plus controversé. Celle que l'on nomme communément The Machine est composée de plus de 8000 étudiants, tous membres des fraternités et sororités blanches implantées sur le campus de l'université. En tant que représentante du système grec traditionnel, son rôle est d'influencer (par des moyens pour le moins douteux) les membres du gouvernement étudiant à voter sur des questions d'ordre spécifique, d'une manière qui lui sera avantageuse. Autrement dit, The Machine « utilise son pouvoir collectif pour diriger le gouvernement étudiant de l'Université de l'Alabama » (p. 50), étouffant du même pas les voix de ceux n'appartenant pas au système grec spécifiquement blanc. L'auteur soutient qu'il s'agit là d' "un symbole concret du privilège blanc » (p. 71) témoignant de la façon insidieuse dont le pouvoir exclusionnaire se déploie sur de nombreux campus américains. Les chapitres 3 et 4 prolongent cette idée et soulignent que ces rapports de domination visent en somme à intimider les étudiants afro-américains en leur adressant le message suivant: «nous sommes les seuls à fixer les normes » (p. 105).

5 Le cheminement des cinquième et sixième chapitres mène le lecteur vers une question fondamentale: d'où vient cette vague de racisme qui déferle actuellement sur les campus? La réponse apportée par l'auteur est sans équivoque: «Le racisme n'a en réalité jamais cessé » (p. 146). C'est précisément sur cette illusion de surprise, poursuit- 
il, que se fondent les réponses timorées des institutions universitaires pour interpréter les actes à caractère raciste recensés sur leur campus. Ces chapitres reviennent donc sur ce qui contribue à développer un sentiment d'hostilité raciale : les appropriations culturelles, les fêtes à thème aux connotations racistes, la pratique du blackface, l'usage du drapeau des Etats confédérés (chapitre 5) ou encore l'emploi de noms controversés pour désigner certains bâtiments universitaires (chapitre 6). L'auteur condamne sans réserve le manque de réactivité et d'anticipation des dirigeants universitaires qui, selon lui, fournissent des "réponses toute faites " (p. 138) contribuant à légitimer et banaliser ce type d'agissements sur les campus.

6 En dépit d'un titre cinglant («We're Mad as Hell... And We're Taking Over the Building »), le septième chapitre conclut l'ouvrage sur une note d'espoir. Au fil de cet ultime développement, Lawrence Ross abandonne sa démarche critique pour suggérer des voies d'action vers une confrontation effective et directe avec le racisme. Parmi ces orientations, l'auteur soumet l'idée d'un congrès national composé d'étudiants afroaméricains qui se réunirait annuellement pour identifier les incidents raciaux sur l'ensemble des campus américains. Cette action collective pourrait, selon l'auteur, " accroître le pouvoir des étudiants noirs, particulièrement au sein des établissements (...) où ils sont sous-représentés " (p. 231). L'auteur fonde également son espoir sur l'activisme étudiant afro-américain qui, depuis son origine au début du $19^{\mathrm{e}}$ siècle, n'a cessé de s'élever contre le maintien du statu quo racial. Sans doute dans un dernier élan d'exaspération, l'auteur va jusqu'à suggérer le boycott par les familles afro-américaines des établissements universitaires qui ne fourniraient pas d'efforts satisfaisants dans la gestion et la prévention du racisme.

$7 \quad \mathrm{Au}$ terme de ce développement nous pouvons toutefois regretter que l'auteur privilégie des voies d'action qui semblent favoriser davantage les réactions offensives que l'écoute et le dialogue au sein de la communauté estudiantine. Plus généralement, on s'étonne de la vision quelque peu monolithique de l'auteur sur les effets du racisme qui pourrait laisser croire que les victimes sont exclusivement afro-américaines. Le racisme, les préjugés et le poids du privilège blanc n'épargnent semble-t-il guère les étudiants issus des minorités hispaniques ou asiatiques. La focalisation de l'ouvrage aurait sans doute mérité d'être élargie, au moins partiellement, de façon à établir un diagnostic plus complet d'une réalité du terrain multidimensionnelle. Malgré ces quelques réserves, Blackballed est un ouvrage nécessaire qui, dans la même veine que Racism without Racists ${ }^{4}$ du sociologue américain Eduardo Bonilla-Silva, montre que le racisme a aujourd'hui migré vers des formes plus subtiles mais tout aussi dévastatrices et avilissantes. Plus largement, ce livre invite le lecteur à se demander si l'on peut censément attendre des universités qu'elles transcendent les sociétés desquelles elles émergent. 


\section{NOTES}

1. Maya Rhodan, «Transcript: Read Full Text of President Barack Obama's Speech in Selma ", Time, 7 mars 2015. Disponible sur : http://time.com/3736357/barack-obama-selma-speechtranscript/

2. Les noms des fraternités et sororités américaines se composent de lettres grecques qui sont souvent les initiales d'une devise grecque ou latine. Pour cette raison, elles sont communément désignées par le terme englobant de « greek letter organizations » ou " GLO » (organisations à lettres grecques). On parle ainsi de « vie grecque ", de "système grec » ou de « communauté grecque ».

3. Lawrence Ross est membre de la fraternité afro-américaine Alpha Phi Alpha.

4. Eduardo Bonilla-Silva, Racism without Racists: Color-Blind Racism and the Persistence of Racial Inequality in America, New York, Rowman \& Littlefield Publishers, 2006.

\section{AUTEURS}

\section{CLÉMENTINE BERTHÉLÉMY}

Aix Marseille Université, LERMA (E.A. 853), Aix-en-Provence 